\title{
Renal Involvement in Leptospirosis - New Insights into Pathophysiology and Treatment
}

\author{
Thaís Bandeira Cerqueira', Daniel Abensur Athanazio ${ }^{2}$, Anne Stambovsky Spichler ${ }^{3,4}$ and Antônio Carlos Seguro ${ }^{4}$ \\ ${ }^{1}$ Medical and Public Health School of Bahia; ${ }^{2}$ Institute of Health Sciences, Federal University of Bahia; Salvador, BA; ${ }^{3}$ Health Municipality \\ Secretariat of São Paulo; ${ }^{4}$ Department of Nephrology - LIM 12, University of São Paulo School of Medicine; São Paulo, SP, Brazil
}

\begin{abstract}
Acute renal failure (ARF) is one of the most common complications of leptospirosis although the causal mechanisms are still unclear. Diverse mechanisms are implicated in leptospiral nephropathy and new data supports the role of peculiar ion transport defects. Besides antibiotic therapy, ARF management in leptospirosis requires dialytic therapy which is most efficient when started early. Dialysis is the standard supportive therapy even though recent evidence suggests clinical benefit from alternative treatments such as plasmapheresis and hemofiltration. Renal recovery is achieved soon after clinical improvement. The comprehension of the primary mechanisms of renal dysfunction will be helpful in the development of additional therapeutic tools for improving supportive therapy for leptospiral nephropathy. This review discusses new insights into mechanisms implicated in leptospiral ARF and recent advances in treatment.
\end{abstract}

Key-Words: Leptospirosis, kidney failure, acute, physiopathology, dialysis.

Leptospirosis is a widespread zoonosis caused by pathogenic spirochetes of the Leptospira spp. genus. Most human infections are followed by asymptomatic seroconversion or undifferentiated self-limited febrile illness. It is not known which factors explain why $5-10 \%$ of all infections present as severe forms requiring hospitalization. Annual incidence of clinical disease is estimated at 10-100 per 100,000 in tropical settings and 0.1-1.0 per 100,000 in temperate regions [1]. In the Sao Paulo metropolitan area, Brazil, the annual incidence of leptospirosis ranged from 1.7 to 2.7 cases per 100,000 inhabitants during the period of 2004-2006 with a fatality rate of $11 \%-18 \%$ [2]. Leptospires colonize renal tubules of a diverse array of mammalian reservoirs which explains the occurrence of leptospirosis in different contexts such as urban outbreaks after floods (due to rat infestation), rural disease in Southeast Asia (transmitted by field mice) and as an occupational disease for those who deal with farm and domestic animals. More recently, leptospirosis reemerged in developed countries associated with water sports or recreational exposure at lakes or canals [3,4]. Human infection occurs after contact with water or soil contaminated by the urine of reservoirs.

Severe forms follow two main patterns: the Weil's triad (acute renal failure, hemorrhagic diathesis and jaundice) or severe pulmonary hemorrhagic syndrome (SPHS). In the Brazilian experience, those forms may present as isolated clinical pictures, for instance, pure Weil's triad which is the typical presentation of leptospirosis in Salvador, Brazil [5] while, in Sao Paulo, most cases of lethal leptospirosis are a combination of both SPHS and Weil's triad [2]. It is still unclear why renal failure predominates as the major cause of death in

Received on 6 December 2007; revised 4 March 2008.

Address for correspondence: Dr Daniel Abensur Athanazio. Departamento de Biointeração - ICS - UFBA. Av. Reitor Miguel Calmon s/n ${ }^{\circ}-$ Campus do Canela. Zip code: 40.110-100 Salvador - Bahia - Brazil. Phone: (71) 3245-8602 Fax: (71) 3240-4194. E-mail: daa@ufba.br.

The Brazilian Journal of Infectious Diseases 2008;12(3):248-252. (C) 2008 by The Brazilian Journal of Infectious Diseases and Contexto Publishing. All rights reserved. some places while pulmonary hemorrhages cause most deaths in others since the most common infecting genomospecies/ serovar is the same in Salvador and São Paulo (Leptospira interrogans serovar Copenhageni) [6,7]. A recent molecular epidemiological study from India suggested that distinct genotype profiles among strains within the same serovar may explain different presentations including pulmonary hemorrhage [8].

Renal involvement in leptospirosis frequently presents as a peculiar picture of hypokalemia and a non-oliguric state. In the last decade, a body of literature has been published supporting distinct mechanisms in the pathophysiology of leptospiral renal disease. Meanwhile, new strategies in supportive care have been suggested. The present work reviews new insights into mechanisms and treatment of acute renal failure (ARF) in leptospirosis.

\section{Clinical Features}

ARF occurs in $16-40 \%$ of human infections with severe symptoms and presentation may range from isolated proteinuria to severe anuric ARF $[3,9,10]$. Non-oliguric forms have better prognosis and oliguria and hyperkalemia have been identified as predictors of a lethal outcome $[6,11,12]$.

Renal failure due to leptospirosis commonly presents as a non-oliguric and a hypokalemic state. Low potassium levels may be detected in $41 \%-45 \%$ of all patients with leptospirosis associated ARF [11,13]. A wasting sodium and potassium defect has been consistently documented by different groups [13-15]. Interestingly, full renal recovery is the rule within two weeks after clinical improvement but an impaired capacity to concentrate urine osmolarity may last for up to 6 months [16].

\section{Pathology}

Renal leptospirosis is usually described as a combination of acute tubular damage and interstitial nephritis. It is not clear, however, whether each lesion is a primary or secondary feature. In a classical description of 33 necropsies, Arean described lesions that ranged in nature and severity as a function of duration of symptoms. Those patients dying within 
one week of illness exhibited acute tubular lesions such as cellular swelling. Those dying between 2-3 weeks of illness presented interstitial edema and acute tubular necrosis (ATN) while those dying after three weeks of symptoms exhibited a diffuse and severe interstitial nephritis [17]. These findings suggest that inflammation is most probably a secondary feature following acute epithelial damage and is observed in patients who survive long enough to develop it. This association between tubular and inflammatory lesions and early/late infection can be easily demonstrated in the hamster model [18]. ATN was recently reported as a usual feature in human necropsies being detected in 13/15 patients from Moldova [15]. It may reflect a direct toxic effect of leptospiral compounds on tubular epithelial cells or indirect effects of dehydration, hypovolemia and ischemia due to ionic wasting defects and the inability to concentrate urine. In animal experimental models, dense colonization by leptospirosis in reservoirs does not result in damage to epithelial cells in direct contact with spirochetes [19]. Thus, any explanation based on toxicity is clouded by our lack of understanding on why the presence of leptospires may cause severe disease or no histopathology in different hosts.

On morphological grounds, it is difficult to address which is the most or first affected nephron segment in leptospirosis. Most works describes a widespread involvement. In an experimental attempt to identify early lesions, distal tubules presented damage before other segments [20] while in human necropsies distal tubules and the ascending limb have been described to be the most severely involved segments [17]. In contrast, other results from human and experimental studies have also reported the most severe lesions to be in proximal segments $[21,22]$. Again, secondary ischemic changes may be superposed with primary lesions directly attributable to leptospires.

\section{Proposed Specific Transport Defects in Tubular Epithelial Cells}

An impaired function of proximal segments was suggested by perfusion studies using microdissected nephrons from guinea pigs. This was based on the observation of a sodium wasting defect paralleling preserved ascending limb function [14]. More recently, two experimental studies in hamster models reported down regulation of the proximal tubule type III $\mathrm{Na}^{+}$/ $\mathrm{H}^{+}$exchanger during acute infection $[18,23]$.

On the other hand, Lin et al. reported a case of Leptospira interrogans serovar Shermani infection from Taiwan with detailed clearance tests. The authors interpreted the absence of glycosuria and of urate and phosphate wasting as evidence of intact proximal tubule function. On the other hand, chloride and osmolar clearances remained unchanged after furosemide infusion [24]. A subsequent study of three patients infected by the same serovar confirmed the clearance tests suggestive of impaired function of the NA, K, 2Cl- cotransporter (NKCC2) of the thick ascending limb (TAL). Such inhibition affects medullar hypertonicity which may induce a defective response to vasopressin. Moreover, the authors demonstrated that protein extracts from the outer membrane of Leptospira santarosai serovar Shermani induced suppression of NKCC2 gene transcription in murine TAL cells [25]. It remains to be clarified whether such findings are reproducible in larger series and whether this is a typical manifestation of serovar Shermani or is shared by all pathogenic leptospires. In an experimental assay to study the role of antibiotics on reversal of tubular transporter defects, the infection by serovar Copenhageni induced both loss of expression of NHE3 in the proximal tubule as well as loss of NKCC2 in TAL which could be prevented by the use of ampicillin in the late stage of infection [18].

Surprisingly, there are few works on clearance tests and detailed characterization of tubular defects of leptospirosis. Such evaluation should be stimulated worldwide in order to expand our comprehension of the exact transport defects in leptospirosis.

Inhibition of $\mathrm{Na}^{+} / \mathrm{K}^{+}$ATPase has been explored as a possible mechanism of tubular defects in leptospirosis. The $\mathrm{Na}^{+} / \mathrm{K}^{+}$pump is the primary source of the sodium gradient which is the driving force that allows potassium reabsorption in proximal tubules and TAL. Glycolipoprotein (GLP) extracts of leptospires inhibit purified $\mathrm{Na}^{+} / \mathrm{K}^{+}$ATPase from rabbit brain and kidneys as well as renal $\mathrm{Na}^{+} / \mathrm{K}^{+}$ATPase from rats, a model of resistance to clinical disease [26]. Microdissection studies detected the inhibitory potential of a standard dose of 350mg of leptospiral GLP along all nephron segments. Convoluted proximal tubules, TAL and distal tubules were the most sensitive to GLP effects and TAL cells may be inhibited by smaller amounts of GLP [26]. This inhibitory effect was postulated to be a primary defect in leptospirosis which could lead to secondary disturbances such as potassium wasting. This effect can be reproduced with fatty acid extracts from leptospiral membranes. Some nonesterified unsaturated fatty acids (NEUFAs) are well established inhibitors of $\mathrm{Na}^{+} / \mathrm{K}^{+}$ ATPase. Indeed, oleic and palmitoleic acids from the leptospiral membrane reproduce such effects [27].

More recently, serum levels of NEUFAs were associated with laboratorial markers of clinical severity in leptospirosis such as creatinine and bilirubin [28]. Although the authors suggest that leptospires may be the primary source of NEUFAs during acute leptospiral infection, it is not clear whether this association reflects toxic effects of leptospires or merely represents the release of NEUFAs by severely affected tissues during an acute disease. The association between NEUFAs and disease severity has also been described in other conditions such as sepsis, trauma and pancreatitis.

All those hypotheses implicating direct effects of leptospires on tubular transporters have a major limitation, that is, their inability to explain why rats or other chronic reservoirs may persist with dense colonization in proximal tubular lumens without any functional impairment. In the case of $\mathrm{Na}^{+} / \mathrm{K}^{+}$ATPase inhibition, rat transporters are also susceptible to GLP effects in microdissection studies. Thus, this single mechanism is not able to explain why the outcomes of infection are different in distinct hosts. 


\section{Role of Pro-Inflammatory Mediators}

Both Toll-like receptors (TLRs) 2 and 4 are constitutively expressed in proximal tubular cells. They are key receptors of innate immunity and potent inducers of the pro-inflammatory response. TLR2 is the major receptor for leptospiral lipopolysaccharides (LPS) [29] while TLR4 recognizes other leptospiral compounds and is implicated in the in vivo control of infection [30]. Exposure of murine proximal tubular cells to outer membrane protein extracts from Leptospira santarosai serovar Shermani results in increased expression of proinflammatory genes by activation of the TLR2 signaling pathway [31]. In vitro studies have already reported activation of a broad range of inflammatory genes such as transcriptional factor NF-êB, inducible nitric oxide synthase (iNOS), monocyte chemotactic protein-1, and tumor necrosis factor a [31-33].

These findings have been postulated to be associated with renal disease as a molecular trigger for interstitial nephritis. Such a connection is hampered by the observation that renal inflammation seems to be a late feature of leptospirosis (discussed above). Furthermore, the impairment in renal function is not associated with the degree of interstitial nephritis in the guinea pig model [22]. In addition, these works use murine cells and it is impossible to predict how these findings mirror the human disease. Interstitial nephritis has not been systematically studied in the mouse model and, indeed, mice are not regarded as an ideal model of experimental leptospirosis since susceptibility or resistance to disease is largely influenced by mouse strain, age, nutritional status and bacterial inoculum [34]. The small amount of data on susceptibility to renal inflammation in mice suggests that there are considerable variations among different mouse strains [35].

On the other hand, in vivo release of pro-inflammatory cytokines may be related to effects other than interstitial nephritis. The observation of increased expression of iNOS in tubular cells after exposure to leptospiral proteins in vitro is of relevance since nitric oxide (NO) is an physiological inhibitor of NKCC2, a postulated target of leptospiral toxins [36]. In the single clinical study evaluation of NO levels in severe leptospirosis, there was a positive correlation between serum NO levels and creatinine [37].

\section{Treatment - The Role of Antibiotics}

The medical literature has no objective data supporting the role of antibiotics in reducing case fatality in leptospirosis. This is probably due to limitations of former trials such as absence of severe involvement $[38,39]$ or underpowered studies due to small samples [40]. A clinical trial in Salvador, Brazil, enrolled 253 patients with late infection (more than 4 days of symptoms) and observed higher mortality in the penicillin treated group (12\%) when compared to the placebo (6\%). Potential adverse effects of treatment, including JarischHerxheimer reaction, were highlighted based on those results but any conclusion may be premature since the observed difference was not significant. The most relevant comparison between penicillin and placebo trials is the observation that treated patients have a shortened period of fever, renal dysfunction and hospitalization [38]. Although there is a consensus that antimicrobial therapy has a major benefit if started within the first 4-5 days of symptoms, the World Health Organization states that most clinicians will treat patients regardless of the period of illness [1].

There is a general belief that after the onset of severe complications such as ARF and SPHS, antimicrobial therapy is unable to improve prognosis and survival depends on adequate supportive therapy. We recently described evidence that, even in late disease, ampicillin may reverse loss of expression of renal transporters (NHE3 and NKCC2) in the hamster model [18].

Recent studies on antimicrobial therapy have focused on the comparison of other antibiotics with standard penicillin therapy. Such evaluations have already been made for ceftriaxone, doxycycline, and cefotaxime $[41,42]$ indicating that all three are effective for severe acute disease. It seems that, from now on, the medical community will probably consider the use of a placebo group in clinical trials of antibiotic therapy in leptospirosis unethical [43].

\section{Treatment - Supportive Therapy and Dialysis}

ARF has been the major cause of death in leptospirosis for a century while in some parts of the world the emergence of SPHS increased the rate of deaths due to pulmonary hemorrhage. Peritoneal dialysis is largely used for the supportive care of leptospirosis-associated ARF worldwide. Recently, a study from Vietnam provided evidence that hemodialysis is superior to peritoneal dialysis in patients with infection-associated ARF (sepsis and malaria) with a higher rate of resolution of acidosis and a faster decline of plasma creatinine levels. In this trial, peritoneal dialysis was associated with more lethal outcomes (47\% x 15\%) and the requirement of more dialytic sessions (70\% x 37\%) [44]. This study was indeed interrupted early due to the magnitude of the differences observed between both groups and suggested that hemodialysis should be the therapy of choice for infectionassociated ARF.

Such conclusions were not accepted by all the scientific community. Some authors highlighted that the method of peritoneal dialysis was probably suboptimal including the choice of rigid catheter, the use of acetate-based replacement fluid, and short dwell time $[45,46]$. The lack of adjustment for severity of illness and other interventions were also emphasized as potential biases of this study [47].

A recent review of the experience of leptospirosisassociated ARF in Thailand showed that blood exchange therapies (hemodialysis, hemofiltration) were associated with lower mortality ( 0 x $10 \%)$, shorter recovery time ( 8 x 16 days) and faster reduction in serum levels of bilirubin, urea and creatinine when compared to standard peritoneal dialysis [48]. It should be noted, however, that there is no data from randomized clinical trials evaluating different methods of renalreplacement therapy in patients with leptospirosis. 
In Emílio Ribas Hospital’s (São Paulo) experience, hemodialysis is the supportive treatment of choice for leptospirosis. A recent comparison was made of the conventional dialysis strategy and sustained low-efficiency dialysis (SLED). In this study a first group was composed of patients who were submitted to dialysis later and realized sessions on alternated days $(\mathrm{n}=15)$. A second group had promptly initiated treatment and were submitted to dialysis daily $(\mathrm{n}=18)$. Conventional hemodialysis (HD) lasted 3 or 4 hours while SLED sessions lasted 6 to 10 hours. As expected, patients from the second group presented serum levels of urea significantly lower than those of the first group (107 \pm 8.2 $\mathrm{mg} / \mathrm{dL}$ vs. $153 \pm 12.4 \mathrm{mg} / \mathrm{dL}$,) and therefore required fewer sessions ( $13 \pm 1.7$ vs. $5.2 \pm 1.7)$. There was no difference in time to recover renal function but death was a more common outcome in the first group (67\% x 17\%). Patients of the second group who recovered also required shorter intervals of intensive care and hospital stay [49]. These data highlight the benefits of dialysis when promptly introduced and performed with longer sessions.

Clinical management also includes vigorous rehydration and special attention to ionic imbalance. Hyperkalemia occurs in severe diseases associated with oliguric forms. In such cases, furosemide is indicated in order to convert it to nonoliguric ARF [50].

\section{Conclusions}

New insights into the pathophysiology and treatment of leptospirois-associated ARF have been published in recent years. A review of classical data and new observations highlights the importance of specific transport defects on renal tubules, which is still under investigation. Interstitial nephritis does not seem to be a primary lesion and works linking in vitro release of cytokines to in vivo disease should be treated with skepticism. Recent observation suggests benefit from blood exchange methods when compared to peritoneal dialysis even though more studies are needed, especially trials comparing different approaches such as hemodialysis, or continuous veno-venous hemofiltration.

\section{References}

1. W.H.O. Human leptospirosis: guidance for diagnosis, surveillance and control. Malta, 2003.

2. Spichler A., Athanazio D., Buzzar M., et al. Using Death Certificate Reports to Find Severe Leptospirosis Cases, Brazil. Emerg Infect Dis 2007;13(10):1559-61.

3. Bharti A.R., Nally J.E., Ricaldi J.N., et al. Leptospirosis: a zoonotic disease of global importance. Lancet Infect Dis 2003;3(12):75771.

4. McBride A.J., Athanazio D.A., Reis M.G. and Ko A.I. Leptospirosis. Curr Opin Infect Dis 2005;18(5):376-86.

5. Costa E., Costa Y.A., Lopes A.A., et al. [Severe forms of leptospirosis: clinical, demographic and environmental aspects]. Rev Soc Bras Med Trop 2001;34(3):261-7.

6. Ko A.I., Galvao Reis M., Ribeiro Dourado C.M., Johnson W.D., Jr. and Riley L.W. Urban epidemic of severe leptospirosis in Brazil. Salvador Leptospirosis Study Group. Lancet 1999;354(9181):820-5.
7. Pereira M.M., Matsuo M.G.S., Bauab A.R., et al. A clonal subpopulation of Leptospira interrogans sensu stricto is the major cause of leptospirosis outbreaks in Brazil. Journal of Clinical Microbiology 2000;38(1):450-2.

8. Natarajaseenivasan K., Vijayachari P., Sharma S., et al. Phylogenetic relatedness among leptospiral strains belonging to same serovar recovered from patients with different clinical syndromes. Infect Genet Evol 2005;5(2):185-91.

9. Visith S., Kearkiat P. Nephropathy in leptospirosis. J Postgrad Med 2005;51(3):184-8

10. Yang H.Y., Hsu P.Y., Pan M.J., et al. Clinical distinction and evaluation of leptospirosis in Taiwan-a case-control study. J Nephrol 2005;18(1):45-53.

11. Lopes A.A., Costa E., Costa Y.A., et al. The association between serum potassium at hospital admission and the case-fatality rate of leptospirosis in men. Revista do Instituto de Medicina Tropical de Sao Paulo 2001;43(4):217-20.

12. Panaphut T., Domrongkitchaiporn S., Thinkamrop B. Prognostic factors of death in leptospirosis: A prospective cohort study in Khon Kaen, Thailand. International Journal of Infectious Diseases 2002;6(1):52-9.

13. Seguro A.C., Lomar A.V., Rocha A.S. Acute renal failure of leptospirosis: nonoliguric and hypokalemic forms. Nephron 1990;55(2):146-51.

14. Magaldi A.J., Yasuda P.N., Kudo L.H., et al. Renal involvement in leptospirosis: a pathophysiologic study. Nephron 1992;62(3):332-9.

15. Covic A., Goldsmith D.J., Gusbeth-Tatomir P., et al. A retrospective 5-year study in Moldova of acute renal failure due to leptospirosis: 58 cases and a review of the literature. Nephrol Dial Transplant 2003;18(6):1128-34.

16. Daher Ede F., Zanetta D.M. and Abdulkader R.C. Pattern of renal function recovery after leptospirosis acute renal failure. Nephron Clin Pract 2004;98(1):c8-14.

17. Arean V.M. The pathologic anatomy and pathogenesis of fatal human leptospirosis (Weil's disease). Amer J Path 1962;40393-423.

18. Spichler A., Ko A.I., Silva E.F., et al. Reversal of renal tubule transporter down-regulation during severe leptospirosis with antimicrobial therapy. Am J Trop Med Hyg 2007;77(6):1111-9.

19. Faine S.B., Adler B., Bolin C. and Perolat P. Leptospira and leptospirosis. 2nd ed. Melbourne, Australia: MediSci, 1999.

20. De Brito T., Freymuller E., Hoshino S. and Penna D.O. Pathology of the kidney and liver in the experimental leptospirosis of the guinea-pig. A light and electron microscopy study. Virchows Arch Pathol Anat Physiol Klin Med 1966;341(1):64-78.

21. de Brito T., Penna D.O., Pereira V.C. and Hoshino S. Kidney biopsies in human leptospirosis: a biochemical and electron microscopy study. Virchows Arch Pathol Anat Physiol Klin Med 1967;343(2):124-35.

22. Davila de Arriaga A.J., Rocha A.S., Yasuda P.H. and De Brito T. Morpho-functional patterns of kidney injury in the experimental leptospirosis of the guinea-pig (L. icterohaemorrhagiae). J Pathol 1982;138(2):145-61.

23. Andrade L., Rodrigues Jr A.C., Sanches T.R., et al. Leptospirosis leads to dysregulation of sodium transporters in the kidney and lung. Am J Physiol Renal Physiol 2006.

24. Lin C.L., Wu M.S., Yang C.W., Huang C.C. Leptospirosis associated with hypokalaemia and thick ascending limb dysfunction [In Process Citation]. Nephrol Dial Transplant 1999;14(1):193-5.

25. Wu M.S., Yang C.W., Pan M.J., et al. Reduced renal Na+-K+-Clco-transporter activity and inhibited NKCC2 mRNA expression by Leptospira shermani: from bed-side to bench. Nephrol Dial Transplant 2004;19(10):2472-9.

26. Younes-Ibrahim M., Buffin-Meyer B., Cheval L., et al. Na,KATPase: a molecular target for Leptospira interrogans endotoxin. Braz J Med Biol Res 1997;30(2):213-23. 
27. Burth P., Younes-Ibrahim M., Goncalez F.H., et al. Purification and characterization of a $\mathrm{Na}^{+}, \mathrm{K}^{+}$ATPase inhibitor found in an endotoxin of Leptospira interrogans. Infect Immun 1997;65(4):1557-60.

28. Burth P., Younes-Ibrahim M., Santos M.C., et al. Role of nonesterified unsaturated fatty acids in the pathophysiological processes of leptospiral infection. J Infect Dis 2005;191(1):51-7.

29. Werts C., Tapping R.I., Mathison J.C., et al. Leptospiral lipopolysaccharide activates cells through a TLR2-dependent mechanism. Nature Immunology 2001;2(4):346-52.

30. Viriyakosol S., Matthias M.A., Swancutt M.A., et al. Toll-Like Receptor 4 Protects against Lethal Leptospira interrogans Serovar Icterohaemorrhagiae Infection and Contributes to In vivo Control of Leptospiral Burden. Infect Immun 2006;74(2):887-95.

31. Yang C.W., Hung C.C., Wu M.S., et al. Toll-like receptor 2 mediates early inflammation by leptospiral outer membrane proteins in proximal tubule cells. Kidney Int 2006;69(5):815-22.

32. Yang C.-W., Wu M.-S., Pan M.-J., et al. Leptospira outer membrane protein activates NF-kappaB and downstream genes expressed in medullary thick ascending limb cells. Journal of the American Society of Nephrology 2000;11(11):2017-26.

33. Yang C.W., Wu M.S., Pan M.J., et al. The Leptospira outer membrane protein LipL32 induces tubulointerstitial nephritismediated gene expression in mouse proximal tubule cells. J Am Soc Nephrol 2002;13(8):2037-45.

34. Faine S. The growth of Leptospira australis B in the kidneys of mice in the incipient experimental carrier state. J Hyg Camb 1962;60:435-42.

35. Athanazio D.A., Santos C.S., Santos A.C., et al. Experimental infection in tumor necrosis factor alpha, interferon gamma and interleukin 4 deficient mice by pathogenic Leptospira interrogans. Acta Tropica 2008;105(1):95-8.

36. Ortiz P.A., Garvin J.L. Role of nitric oxide in the regulation of nephron transport. Am J Physiol Renal Physiol 2002;282(5):F777-84.

37. Maciel E.A., Athanazio D.A., Reis E.A., et al. High serum nitric oxide levels in patients with severe leptospirosis. Acta Trop 2006;100(3):256-60.
38. Watt G., Padre L.P., Tuazon M.L., et al. Placebo-controlled trial of intravenous penicillin for severe and late leptospirosis. Lancet 1988; $1(8583)$ : 433-5.

39. Edwards C.N., Nicholson G.D., Hassell T.A., et al. Penicillin therapy in icteric leptospirosis. Am J Trop Med Hyg 1988;39(4):38890.

40. Daher E., Nogueira C.B. Evaluation of penicillin therapy in patients with leptospirosis and acute renal failure. Rev Inst Med Trop Sao Paulo 2000;42(6):327-32.

41. Panaphut T., Domrongkitchaiporn S., Vibhagool A., et al. Ceftriaxone compared with sodium penicillin $\mathrm{g}$ for treatment of severe leptospirosis. Clin Infect Dis 2003;36(12):1507-13.

42. Suputtamongkol Y., Niwattayakul K., Suttinont C., et al. An open, randomized, controlled trial of penicillin, doxycycline, and cefotaxime for patients with severe leptospirosis. Clin Infect Dis 2004;39(10):1417-24.

43. Vinetz J.M. A mountain out of a molehill: do we treat acute leptospirosis, and if so, with what? Clin Infect Dis 2003;36(12):1514-5.

44. Phu N.H., Hien T.T., Mai N.T., et al. Hemofiltration and peritoneal dialysis in infection-associated acute renal failure in Vietnam. $\mathrm{N}$ Engl J Med 2002;347(12):895-902.

45. Daugirdas J.T. Peritoneal dialysis in acute renal failure-why the bad outcome? N Engl J Med 2002;347(12):933-5.

46. Rao P.S., Modi K.S. Hemofiltration and peritoneal dialysis in infection-associated acute renal failure. $N$ Engl $\mathrm{J}$ Med 2003;348(9):858-60.

47. Casserly L.F. Hemofiltration and peritoneal dialysis in infection-associated acute renal failure. N Engl J Med 2003;348(9):858-60.

48. Wiwanitkit V. Comparison between blood exchange and classical therapy for acute renal failure in Weil's disease: appraisal on Thai reports. Nephrology (Carlton) 2006;11(5):481.

49. Andrade L., Cleto S., Seguro A.C. Door-to-dialysis time and daily hemodialysis in patients with leptospirosis: impact on mortality. Clin J Am Soc Nephrol 2007;2(4):739-44.

50. Niwattayakul K., Sitprija V. Leptospiral acute renal failure: effects of dopamine and furosemide. Ren Fail 2007;29(2):159-62. 